\title{
Numerical Algorithm to Solve a Class of Variable Order Fractional Integral-Differential Equation Based on Chebyshev Polynomials
}

\author{
Kangwen Sun and Ming Zhu \\ School of Aeronautic Science and Technology, Beihang University, Beijing 100191, China \\ Correspondence should be addressed to Ming Zhu; zhuming9091@163.com
}

Received 24 May 2015; Revised 30 August 2015; Accepted 31 August 2015

Academic Editor: Kishin Sadarangani

Copyright @ $2015 \mathrm{~K}$. Sun and M. Zhu. This is an open access article distributed under the Creative Commons Attribution License, which permits unrestricted use, distribution, and reproduction in any medium, provided the original work is properly cited.

\begin{abstract}
The purpose of this paper is to study the Chebyshev polynomials for the solution of a class of variable order fractional integraldifferential equation. The properties of Chebyshev polynomials together with the four kinds of operational matrixes of Chebyshev polynomials are used to reduce the problem to the solution of a system of algebraic equations. By solving the algebraic equations, the numerical solutions are acquired. Further some numerical examples are shown to illustrate the accuracy and reliability of the proposed approach and the results have been compared with the exact solution.
\end{abstract}

\section{Introduction}

Fractional calculus has attracted increasing attention for decades since it plays a vital role in different disciplines of science and engineering [1-3]. Compared with integer order differential equation, fractional differential equation has the advantage that it can better describe some natural physics processes and dynamic system processes, because the fractional order differential operators are nonlocal operators. Many physics, chemistry, and engineering systems can be elegantly modeled with the help of the FDEs, such as dielectric polarization, viscoelastic systems, control theory, chaotic behavior, and electrolyte-electrolyte polarization [46]. Since its tremendous applications in several disciplines, considerable attention has been given to the exact and the numerical solutions of fractional differential equations and fractional integral equations. Even numerical approximation of fractional differentiation of rough functions is not easy as it is an ill-posed problem.

Other than modeling aspects of these differential equations, the solution techniques and their reliability are rather more significant. In order to obtain the goal of highly accurate and reliable solutions, several methods have been proposed to solve the fractional order differential and fractional order integral equations. The most commonly used methods are
Variational Iteration Method [7], Adomian Decomposition Method [8, 9], Generalized Differential Transform Method $[10,11]$, and Wavelet Method [12, 13].

Recently, more and more physicists and mathematicians are finding that numerous important dynamical problems exhibit fractional order behavior which can vary with space and time. This fact indicates that variable order calculus provides an effective mathematical framework for the description of complex dynamical problems. The concept of a variable order operator is a much more recent development, which is a new orientation in engineering. Many researchers have proposed different definitions of variable order differential operators, each of these with a specific meaning to get desired goals. The variable order operator definitions recently proposed in the engineering include the RiemannLiouville definition, Marchaud definition, Grünwald definition, Caputo definition, and Coimbra definition $[14,15]$.

In this paper, the main objective is to introduce the Chebyshev polynomials method to solve the variable order fractional integral-differential equation. The method is based on reducing the equation to a system of algebraic equations by expanding the solution as Chebyshev polynomials with unknown coefficients. The main characteristic of an operational method is to convert the integral-differential equation 
into an algebraic one. It not only simplifies the problem but also speeds up the computation.

Our study focuses on a class of variable order fractional integral-differential equation as follows:

$$
\begin{gathered}
D^{\alpha(t)}[u(x, t) g(x, t)]+\frac{\partial u(x, t)}{\partial t}+\int_{0}^{t} u(x, T) d T \\
+\int_{0}^{t} u(x, T) k(x, T) d T=f(x, t)
\end{gathered}
$$

subject to the initial conditions

$$
\begin{gathered}
u(x, 0)=g(x) \quad x \in[0,1], \\
u(0, t)=h(t) \quad t \in[0,1],
\end{gathered}
$$

where $D^{\alpha(x)}(u(x, t) g(x, t))$ is fractional derivative of Caputo sense; when $g(x, t)=u(x, t)$, the initial problem is changed to nonlinear equation. $f(x, t), g(x, t), u(x, t)$, and $k(x, t)$ are assumed to be casual functions of time and space on the section $(x, t) \in[0,1] \times[0,1]$, where $f(x, t), g(x, t)$, and $k(x, t)$ are known and $u(x, t)$ is the unknown, $0<\alpha(x) \leq 1$.

\section{Chebyshev Polynomials and Their Properties}

The well-known Chebyshev polynomials are defined on the interval $[-1,1]$ and can be determined with the recurrence formula [16]

$$
\begin{aligned}
\widehat{T}_{n+1}(x)=2 x \widehat{T}_{n}(x)-\widehat{T}_{n-1}(x), \\
\widehat{T}_{0}(x)=1, \widehat{T}_{1}(x)=x, n=1,2, \ldots
\end{aligned}
$$

The analytic form of the Chebyshev polynomials $\widehat{T}_{n}(x)$ of degree $n$ is given by

$$
\widehat{T}_{n}(x)=n \sum_{i=0}^{[n / 2]}(-1)^{i} 2^{n-2 i-1} \frac{(n-i-1) !}{(i) !(n-2 i) !} x^{n-2 i}
$$

where $[n / 2]$ denotes the integer part of $n / 2$ and $n$ denotes positive integer. The orthogonality condition is

$$
\int_{-1}^{1} \frac{\widehat{T}_{i}(x) \widehat{T}_{j}(x)}{\sqrt{1-x^{2}}} d x= \begin{cases}\pi, & i=j=0 \\ \frac{\pi}{2}, & i=j \neq 0 \\ 0, & i \neq j .\end{cases}
$$

In order to use these polynomials on the interval $[0,1]$, we define the shifted Chebyshev polynomials by introducing the change of variable $x=2 t-1$. Therefore, the shifted Chebyshev polynomials are defined as $T_{n}^{*}(t)=\widehat{T}_{n}(2 t-1)$. The analytic form of the shifted Chebyshev polynomials $T_{n}^{*}(t)$ of degree $n$ is given by

$$
T_{n}^{*}(t)=n \sum_{k=0}^{n}(-1)^{n-k} \frac{2^{2 k}(n+k-1) !}{(2 k) !(n-k) !} t^{k}, \quad n=1,2, \ldots
$$

Let

$$
\Phi(t)=\left[T_{0}^{*}(t), T_{1}^{*}(t), \ldots, T_{n}^{*}(t)\right]^{T}
$$

The Chebyshev polynomials given by (6) can be expressed in the matrix form

where

$$
\Phi(t)=\mathbf{A T}_{n}(t)
$$

$$
\begin{aligned}
& \mathbf{A}=\left[\begin{array}{ccccc}
1 & 0 & 0 & \cdots & 0 \\
-1 & 2 & 0 & \cdots & 0 \\
2(-1)^{2} \frac{1 !}{2 !} & 2(-1)^{1} \frac{2^{2} 2 !}{2 !} & 2(-1)^{0} \frac{2^{4} 3 !}{4 !} & \cdots & 0 \\
\vdots & \vdots & \vdots & \ddots & \vdots \\
n(-1)^{n} \frac{(n-1) !}{n !} & n(-1)^{n-1} \frac{2^{2} n !}{2 !(n-1) !} & n(-1)^{n-2} \frac{2^{4}(n+1) !}{4 !(n-2) !} & \cdots & n(-1)^{0} \\
\frac{2^{2 n}(2 n-1) !}{(2 n) !}
\end{array}\right], \\
& \mathbf{T}_{n}(t)=\left[\begin{array}{c}
1 \\
t \\
\vdots \\
t^{n}
\end{array}\right] .
\end{aligned}
$$


Obviously

$$
\mathbf{T}_{n}(t)=\mathbf{A}^{-1} \boldsymbol{\Phi}(t)
$$

A function $u(t) \in L^{2}(0,1)$ can be expressed in terms of the Chebyshev basis. In practice, only the first $(n+1)$ term of Chebyshev polynomials is considered. Hence

$$
u(t) \cong \sum_{i=0}^{n} c_{i} T_{i}^{*}(t)=c^{T} \Phi(t)
$$

where $\mathbf{c}=\left[c_{0}, c_{1}, \ldots, c_{n}\right]^{T}, c_{i}(i=0,1,2, \ldots, n)$ are called Chebyshev coefficients and $\mathbf{c}=\mathbf{Q}^{-1}(u, \Phi(t))$. The dimension of $\mathbf{Q}$ is $(n+1) \times(n+1)$; it is called the inner product matrix which is given by

$$
\begin{aligned}
\mathbf{Q} & =\int_{0}^{1} \boldsymbol{\Phi}(t) \boldsymbol{\Phi}^{T}(t) d x=\int_{0}^{1}\left(\mathbf{A T}_{n}(t)\right)\left(\mathbf{A T}_{n}(t)\right)^{T} d t \\
& =\mathbf{A}\left(\int_{0}^{1} \mathbf{T}_{n}(t) \mathbf{T}_{n}^{T}(t) d t\right) \mathbf{A}^{T}=\mathbf{A H A}^{T},
\end{aligned}
$$

where

$$
\mathbf{H}=\left[\begin{array}{cccc}
1 & \frac{1}{2} & \cdots & \frac{1}{n+1} \\
\frac{1}{2} & \frac{1}{3} & \cdots & \frac{1}{n+2} \\
\vdots & \vdots & \ddots & \vdots \\
\frac{1}{n+1} & \frac{1}{n+2} & \cdots & \frac{1}{2 n+1}
\end{array}\right]
$$

For the function $u(x, t) \in L^{2}([0,1] \times[0,1])$, we can also obtain its approximation by using Chebyshev polynomials

$$
u(x, t) \cong \sum_{i=0}^{n} \sum_{j=0}^{n} u_{i j} T_{i}^{*}(t) T_{j}^{*}(t)=\boldsymbol{\Phi}^{T}(x) \mathbf{U} \boldsymbol{\Phi}(t),
$$

where

$$
\mathbf{U}=\left[\begin{array}{cccc}
u_{00} & u_{01} & \cdots & u_{0 n} \\
u_{10} & u_{11} & \cdots & u_{1 n} \\
\vdots & \vdots & \ddots & \vdots \\
u_{n 0} & u_{n 1} & \cdots & u_{n n}
\end{array}\right]
$$

Theorem 1 (see [16]). The error in approximating $u(t)$ by the sum of its first $n$ terms is bounded by the sum of the absolute values of all the neglected coefficients. If

$$
u_{n}(t)=\sum_{i=0}^{n} c_{i} T_{i}^{*}(t)
$$

then

$$
E_{T}(m)=\left|u(t)-u_{n}(t)\right| \leq \sum_{k=n+1}^{\infty}\left|c_{k}\right|
$$

for all $u(t)$, all $n$, and all $t \in[-1,1]$.
Theorem 2 (see [17]). The Caputo fractional derivative of order $\alpha$ for the shifted Chebyshev polynomials can be expressed in terms of the shifted Chebyshev polynomials themselves in the following form:

$$
D^{\alpha}\left(T_{i}^{*}(t)\right)=\sum_{k=\lceil\alpha\rceil}^{i} \sum_{j=0}^{k-\lceil\alpha\rceil} \theta_{i, j, k} T_{j}^{*}(t)
$$

where

$$
\begin{array}{r}
\theta_{i, j, k} \\
=\frac{(-1)^{i-k} 2 i(i+k-1) ! \Gamma(k-\alpha+1 / 2)}{\Gamma(k+1 / 2)(i-k) ! \Gamma(k-\alpha-j+1) \Gamma(k-\alpha+j+1)}, \\
j=0,1, \ldots .
\end{array}
$$

Theorem 3. The error $E_{T}^{*}(m)=\left|D^{\alpha}(u(t))-D^{\alpha}\left(u_{n}(t)\right)\right|$ in approximating $D^{\alpha}(u(t))$ by $D^{\alpha}\left(u_{n}(t)\right)$ is bounded by $\left|E_{T}^{*}(m)\right| \leq$ $\left|\sum_{i=n+1}^{\infty} c_{i}\left(\sum_{k=\lceil\alpha\rceil}^{i} \sum_{j=0}^{k-\lceil\alpha\rceil} \theta_{i, j, k}\right)\right|$.

Proof. A combination of (17) and (25) leads to

$$
\begin{aligned}
\left|E_{T}^{*}(m)\right| & =\left|D^{\alpha}(u(t))-D^{\alpha}\left(u_{n}(t)\right)\right| \\
& =\left|\sum_{i=n+1}^{\infty} c_{i}\left(\sum_{k=\lceil\alpha\rceil}^{i} \sum_{j=0}^{k-\lceil\alpha\rceil} \theta_{i, j, k} T_{j}^{*}(t)\right)\right|,
\end{aligned}
$$

but $\left|T_{j}^{*}\right| \leq 1$, so we can get

$$
\left|E_{T}^{*}(m)\right| \leq\left|\sum_{i=n+1}^{\infty} c_{i}\left(\sum_{k=\lceil\alpha\rceil}^{i} \sum_{j=0}^{k-\lceil\alpha\rceil} \theta_{i, j, k}\right)\right|,
$$

subtracting the truncated series from the infinite series, bounding each term in the difference, summing the bounds, and hence completing the proof of the theorem.

\section{Operational Matrix of the Chebyshev Polynomials}

3.1. Fractional Calculus. Before we introduce the Chebyshev polynomials operational matrix of the fractional integration, we first review some basic definitions of fractional calculus, which have been given in [18].

Definition 4. The Riemann-Liouville fractional integral operator of order $\alpha(t)$ :

$$
\begin{array}{r}
I_{a+}^{\alpha(t)} u(t)=\frac{1}{\Gamma(\alpha(t))} \int_{a+}^{t}(t-T)^{\alpha(t)-1} u(T) d T, \\
t>0[\operatorname{Re}(\alpha(t))>0] .
\end{array}
$$

Definition 5. Riemann-Liouville fractional derivate with order $\alpha(t)$ :

$$
D_{a+}^{\alpha(t)} u(t)=\frac{1}{\Gamma(m-\alpha(t))} \frac{d^{m}}{d t^{m}} \int_{a}^{t} \frac{u(\tau)}{(t-\tau)^{\alpha(t)-m+1}} d \tau
$$


Definition 6. Caputo's fractional derivate with order $\alpha(t)$, $(0<\alpha(t) \leq 1)$

$$
\begin{aligned}
D^{\alpha(t)} u(t)= & \frac{1}{\Gamma(1-\alpha(t))} \int_{0+}^{t}(t-\tau)^{-\alpha(t)} u^{\prime}(\tau) d \tau \\
& +\frac{(u(0+)-u(0-)) t^{-\alpha(t)}}{\Gamma(1-\alpha(t))} .
\end{aligned}
$$

If we assume the starting time in a perfect situation, we can obtain the definition as follows:

$$
\begin{array}{r}
D^{\alpha(t)} u(t)=\frac{1}{\Gamma(1-\alpha(t))} \int_{0+}^{t}(t-\tau)^{-\alpha(t)} u^{\prime}(\tau) d \tau \\
\quad(0<\alpha(t)<1) .
\end{array}
$$

Generally, we adopt (25) as the definition of fractional derivate in Caputo sense. With the definition above, we can obtain the following formula $(0<\alpha(t) \leq 1)$ :

$$
\begin{aligned}
& D^{\alpha(t)} c=0 \\
& D^{\alpha(t)} x^{\beta} \\
& \quad= \begin{cases}0 & \beta=0 \\
\frac{\Gamma(\beta+1)}{\Gamma(\beta+1-\alpha(t))} x^{\beta-a(t)} & \beta=1,2,3, \ldots .\end{cases}
\end{aligned}
$$

3.2. The Operational Matrix of the Section as $\partial u(x, t) / \partial t$ in terms of Chebyshev Polynomials. The differentiation of vector $\Phi(t)$ in (7) can be given by

$$
\Phi^{\prime}(t)=\mathbf{D} \Phi(t)
$$

where $\mathbf{D}$ is the $(n+1) \times(n+1)$ operational matrix of derivatives for Chebyshev polynomials. From (8) we have

$$
\boldsymbol{\Phi}^{\prime}(t)=\mathbf{A}\left[\begin{array}{c}
0 \\
1 \\
\vdots \\
n t^{n-1}
\end{array}\right] \text {. }
$$

Define the $(n+1) \times(n)$ matrix $\mathbf{V}_{(n+1) \times n}$ and vector $\mathbf{T}_{n}^{*}(t)$ as

$$
\mathbf{V}_{(n+1) \times n}=\left[\begin{array}{cccc}
0 & 0 & \cdots & 0 \\
1 & 0 & \cdots & 0 \\
0 & 2 & \cdots & 0 \\
\vdots & \vdots & \ddots & \vdots \\
0 & 0 & \cdots & n
\end{array}\right] \text {, }
$$

$$
\mathbf{T}_{n}^{*}(t)=\left[\begin{array}{c}
1 \\
t \\
\vdots \\
t^{n-1}
\end{array}\right]_{(n \times 1)} .
$$

Equation (28) may then be restated as

$$
\boldsymbol{\Phi}^{\prime}(t)=\mathbf{A} \mathbf{V}_{(n+1) \times n} \mathbf{T}_{n}^{*}(t)
$$

Now we expand vector $\mathbf{T}_{n}^{*}(t)$ in terms of $\boldsymbol{\Phi}(t)$. From (10), we get

$$
\mathbf{T}_{n}^{*}(t)=\mathbf{B}^{*} \boldsymbol{\Phi}(t)
$$

where

$$
\mathbf{B}^{*}=\left[\mathbf{A}_{[1]}^{-1}, \mathbf{A}_{[2]}^{-1}, \ldots, \mathbf{A}_{[n]}^{-1}\right]^{T} .
$$

$\mathbf{A}_{[k]}^{-1}$ is $k$ th row of $\mathbf{A}^{-1}, k=1,2, \ldots, n$.

Then we have

$$
\boldsymbol{\Phi}^{\prime}(t)=\mathbf{A V}_{(n+1) \times n} \mathbf{B}^{*} \boldsymbol{\Phi}(t) .
$$

Therefore we obtain the operational matrix of the section as $\partial u(x, t) / \partial t$ as follows:

$$
\begin{aligned}
\frac{\partial u(x, t)}{\partial t} & =\Phi^{T}(x) \mathbf{U} \Phi^{\prime}(t) \\
& =\Phi^{T}(x) \mathbf{U A V}_{(n+1) \times n} \mathbf{B}^{*} \boldsymbol{\Phi}(t)
\end{aligned}
$$

3.3. The Operational Matrix of the Section as $D^{\alpha(t)}(u(x$, t) $g(x, t))$ in terms of Chebyshev Polynomials. If we approximate the functions $u(x, t), g(x, t)$ with Chebyshev polynomials, they can be written as $u(x, t)=\Phi^{T}(x) \mathbf{U} \Phi(t)$ and $g(x, t)=\boldsymbol{\Phi}^{T}(x) \mathbf{G} \boldsymbol{\Phi}(t)$, where $\mathbf{U}$ is unknown and $\mathbf{G}$ is known. Then we have

$$
\begin{aligned}
D^{\alpha(t)} & {[u(x, t) g(x, t)]=D^{\alpha(t)}\left[\boldsymbol{\Phi}^{T}(x) \mathbf{U} \boldsymbol{\Phi}(t) \boldsymbol{\Phi}^{T}(t) \mathbf{G} \boldsymbol{\Phi}(x)\right]=\boldsymbol{\Phi}^{T}(x) \mathbf{U} D^{\alpha(t)}\left[\boldsymbol{\Phi}(t) \boldsymbol{\Phi}^{T}(t)\right] \mathbf{G} \boldsymbol{\Phi}(x) } \\
= & \boldsymbol{\Phi}^{T}(x) \mathbf{U} D^{\alpha(t)}\left[\mathbf{A T}_{n}^{*}(t)\left(\mathbf{A T}_{n}^{*}(t)\right)^{T}\right] \mathbf{A}^{T} \mathbf{G} \boldsymbol{\Phi}(x)=\boldsymbol{\Phi}^{T}(x) \mathbf{U A} D^{\alpha(t)}\left[\mathbf{T}_{n}^{*}(t)\left(\mathbf{T}_{n}^{*}(t)\right)^{T}\right] \mathbf{A}^{T} \mathbf{G} \boldsymbol{\Phi}(x) \\
& =\boldsymbol{\Phi}^{T}(x) \mathbf{U A} D^{\alpha(t)}\left(\left[\begin{array}{c}
1 \\
t \\
\vdots \\
t^{n}
\end{array}\right]\left[\begin{array}{llll}
1 & t & \cdots & t^{n}
\end{array}\right] \mathbf{A}^{T} \mathbf{G} \boldsymbol{\Phi}(x)=\boldsymbol{\Phi}^{T}(x) \mathbf{U A} D^{\alpha(t)}\left[\begin{array}{cccc}
1 & t & \cdots & t^{n} \\
t & t^{2} & \cdots & t^{n+1} \\
\vdots & \vdots & \ddots & \vdots \\
t^{n} & & & \\
t^{2 n} & \cdots & t^{2 n}
\end{array}\right] \mathbf{A}^{T} \mathbf{G} \boldsymbol{\Phi}(x)\right.
\end{aligned}
$$




$$
\begin{aligned}
& =\boldsymbol{\Phi}^{T}(x) \mathbf{U A}\left[\begin{array}{cccc}
0 & \frac{\Gamma(2)}{\Gamma(2-\alpha(t))} t^{1-\alpha(t)} & \cdots & \frac{\Gamma(n+1)}{\Gamma(n+1-\alpha(t))} t^{n-\alpha(t)} \\
\frac{\Gamma(2)}{\Gamma(2-\alpha(t))} t^{1-\alpha(t)} & \frac{\Gamma(3)}{\Gamma(3-\alpha(t))} t^{2-\alpha(t)} & \cdots & \frac{\Gamma(n+2)}{\Gamma(n+2-\alpha(t))} t^{n+1-\alpha(t)} \\
\vdots & \vdots & \ddots & \vdots \\
\frac{\Gamma(n+1)}{\Gamma(n+1-\alpha(t))} t^{n-\alpha(t)} & \frac{\Gamma(n+2)}{\Gamma(n+2-\alpha(t))} t^{n+1-\alpha(t)} & \cdots & \frac{\Gamma(2 n+1)}{\Gamma(2 n+1-\alpha(t))} t^{2 n-\alpha(t)}
\end{array}\right] \mathbf{A}^{T} \mathbf{G} \boldsymbol{\Phi}(x) \\
& =\Phi^{T}(x) \text { UAMA }^{T} \mathbf{G} \Phi(x) \text {. }
\end{aligned}
$$

Now we define

$$
\mathbf{M}=\left[\begin{array}{cccc}
0 & \frac{\Gamma(2)}{\Gamma(2-\alpha(t))} t^{1-\alpha(t)} & \cdots & \frac{\Gamma(n+1)}{\Gamma(n+1-\alpha(t))} t^{n-\alpha(t)} \\
\frac{\Gamma(2)}{\Gamma(2-\alpha(t))} t^{1-\alpha(t)} & \frac{\Gamma(3)}{\Gamma(3-\alpha(t))} t^{2-\alpha(t)} & \cdots & \frac{\Gamma(n+2)}{\Gamma(n+2-\alpha(t))} t^{n+1-\alpha(t)} \\
\vdots & \vdots & \ddots & \vdots \\
\frac{\Gamma(n+1)}{\Gamma(n+1-\alpha(t))} t^{n-\alpha(t)} & \frac{\Gamma(n+2)}{\Gamma(n+2-\alpha(t))} t^{n+1-\alpha(t)} & \cdots & \frac{\Gamma(2 n+1)}{\Gamma(2 n+1-\alpha(t))} t^{2 n-\alpha(t)}
\end{array}\right] .
$$

$\mathbf{M}$ is called the operational matrix of the section as $D^{\alpha(t)}(u(x$, t) $g(x, t))$ with Chebyshev polynomials.

So we have

$$
D^{\alpha(t)}[u(x, t) g(x, t)]=\Phi^{T}(x) \mathbf{U A M A}^{T} \mathbf{G} \Phi(x) .
$$

3.4. The Operational Matrix of the Section as $\int_{0}^{t} u(x, T) d T$ in terms of Chebyshev Polynomials. The integration of the vector $\Phi(t)$ in (7) can be expressed as

$$
\int_{0}^{t} \boldsymbol{\Phi}(t) d T=\mathbf{P} \boldsymbol{\Phi}(t)
$$

where $\mathbf{P}$ is the $(n+1) \times(n+1)$ operational matrix of integration for Chebyshev polynomials. So we have

$$
\begin{aligned}
\int_{0}^{t} \boldsymbol{\Phi}(T) d T & =\int_{0}^{t} \mathbf{A T}_{n}(T) d T=\mathbf{A} \int_{0}^{t} \mathbf{T}_{n}(T) d T \\
& =\mathbf{A} \int_{0}^{t}\left[\begin{array}{c}
c 1 \\
T \\
\vdots \\
T^{n}
\end{array}\right] d T=\mathbf{A}\left[\begin{array}{c}
t \\
\frac{1}{2} t^{2} \\
\vdots \\
\frac{1}{n+1} t^{n+1}
\end{array}\right] \\
& =\mathbf{A}_{p} \mathbf{T}_{p},
\end{aligned}
$$

$$
\text { where } \mathbf{A}_{p} \text { is an }(n+1) \times(n+1) \text { matrix: }
$$

$$
\begin{gathered}
\mathbf{A}_{p}=\mathbf{A}\left[\begin{array}{cccc}
1 & 0 & \cdots & 0 \\
0 & \frac{1}{2} & \cdots & 0 \\
\vdots & \vdots & \ddots & \vdots \\
0 & 0 & \cdots & \frac{1}{n+1}
\end{array}\right], \\
\mathbf{T}_{p}=\left[\begin{array}{c}
t \\
t^{2} \\
\vdots \\
t^{n+1}
\end{array}\right]
\end{gathered}
$$

Now we approximate the elements of vector $\mathbf{T}_{p}$ in terms of $\Phi(\mathrm{t})$. By (10), then we have

$$
t^{k}=\mathbf{A}_{[k+1]}^{-1} \boldsymbol{\Phi}(t), \quad k=1, \ldots, n,
$$

where $\mathbf{A}_{[k+1]}^{-1}$ is the $k+1$ th row of $\mathbf{A}^{-1}$ for $k=1, \ldots, n$. We just need to approximate $t^{n+1}=\mathbf{c}_{n+1}^{T} \Phi(t)$. By using $\mathbf{c}=$ $\mathbf{Q}^{-1}(u, \Phi(t))$, we have 


$$
\begin{aligned}
& \mathbf{c}_{n+1}=\mathbf{Q}^{-1} \int_{0}^{1} t^{n+1} \boldsymbol{\Phi}(t) d t \\
& =\mathbf{Q}^{-1}\left[\begin{array}{c}
\int_{0}^{1} t^{n+1} B_{0, n}(t) d t \\
\int_{0}^{1} t^{n+1} B_{1, n}(t) d t \\
\vdots \\
\int_{0}^{1} t^{n+1} B_{n, n}(t) d t
\end{array}\right] \\
& =\frac{\mathbf{Q}^{-1}}{2 n+2}\left[\frac{\left(\begin{array}{c}
n \\
0
\end{array}\right)}{\left(\begin{array}{c}
2 n+1 \\
n+1
\end{array}\right)} \frac{\left(\begin{array}{c}
n \\
1
\end{array}\right)}{\left(\begin{array}{c}
2 n+1 \\
n+2
\end{array}\right)} \cdots \frac{\left(\begin{array}{c}
n \\
n
\end{array}\right)}{\left(\begin{array}{c}
2 n+1 \\
2 n+1
\end{array}\right)}\right]^{T} \text {. }
\end{aligned}
$$

We define

$$
\mathbf{P}_{1}=\left[\begin{array}{c}
\mathbf{A}_{[2]}^{-1} \\
\mathbf{A}_{[3]}^{-1} \\
\vdots \\
\mathbf{A}_{[n+1]}^{-1} \\
\mathbf{c}_{n+1}
\end{array}\right]
$$

Then we can get $\mathbf{T}_{p}=\mathbf{P}_{1} \boldsymbol{\Phi}(t)$. Therefore we have the operational matrix of integration as follows:

$$
\mathbf{P}=\mathbf{A}_{p} \mathbf{P}_{1}
$$

So we have

$$
\begin{aligned}
\int_{0}^{t} u(x, T) d T & =\int_{0}^{t} \boldsymbol{\Phi}^{T}(x) \mathbf{U} \boldsymbol{\Phi}(T) d T \\
& =\boldsymbol{\Phi}^{T}(x) \mathbf{U} \int_{0}^{t} \boldsymbol{\Phi}(T) d T \\
& =\boldsymbol{\Phi}^{T}(x) \mathbf{U P} \boldsymbol{\Phi}(t) \\
& =\boldsymbol{\Phi}^{T}(x) \mathbf{U A}_{p} \mathbf{P}_{1} \boldsymbol{\Phi}(t)
\end{aligned}
$$

3.5. The Operational Matrix of the Section as $\int_{0}^{t} u(x, T) k(x$, T)dT in terms of Chebyshev Polynomials. Firstly, we approximate the function $k(x, t)$ with Chebyshev polynomials; it can be written as $k(x, t)=\Phi^{T}(x) \mathbf{K} \Phi(t)$, and $\mathbf{K}$ is known. So we have

$$
\begin{aligned}
& \int_{0}^{t} u(x, T) k(x, t) d T \\
& \quad=\int_{0}^{t}\left(\boldsymbol{\Phi}^{T}(x) \mathbf{U} \boldsymbol{\Phi}(T) \boldsymbol{\Phi}^{T}(T) \mathbf{K} \boldsymbol{\Phi}(x)\right) d T=\boldsymbol{\Phi}^{T}(x) \\
& \cdot \mathbf{U} \int_{0}^{t}\left(\boldsymbol{\Phi}(T) \boldsymbol{\Phi}^{T}(T)\right) d T \mathbf{K} \boldsymbol{\Phi}(x)=\boldsymbol{\Phi}^{T}(x)
\end{aligned}
$$

$$
\begin{aligned}
& \cdot \mathbf{U A} \int_{0}^{t}\left[\begin{array}{cccc}
1 & T & \cdots & T^{n} \\
T & T^{2} & \ldots & T^{n+1} \\
\vdots & \vdots & \ddots & \vdots \\
T^{n} & T^{n+1} & \cdots & T^{2 n}
\end{array}\right] d T \mathbf{A}^{T} \mathbf{K} \boldsymbol{\Phi}(x)=\boldsymbol{\Phi}^{T}(x) \\
& \cdot\left[\begin{array}{cccc}
t & \frac{1}{2} t^{2} & \cdots & \frac{1}{n+1} t^{n+1} \\
\frac{1}{2} t^{2} & \frac{1}{3} t^{3} & \cdots & \frac{1}{n+2} t^{n+2} \\
\vdots & \vdots & \ddots & \vdots \\
\frac{1}{n+1} t^{n+1} & \frac{1}{n+2} t^{n+2} & \cdots & \frac{1}{2 n+1} t^{2 n+1}
\end{array}\right] \mathbf{A}^{T} \mathbf{K} \boldsymbol{\Phi}(x) \\
& =\boldsymbol{\Phi}^{T}(x) \mathbf{U A R A} \mathbf{H A}^{T} \mathbf{K}(x) .
\end{aligned}
$$

We define

$$
\mathbf{R}=\left[\begin{array}{cccc}
t & \frac{1}{2} t^{2} & \cdots & \frac{1}{n+1} t^{n+1} \\
\frac{1}{2} t^{2} & \frac{1}{3} t^{3} & \cdots & \frac{1}{n+2} t^{n+2} \\
\vdots & \vdots & \ddots & \vdots \\
\frac{1}{n+1} t^{n+1} & \frac{1}{n+2} t^{n+2} & \cdots & \frac{1}{2 n+1} t^{2 n+1}
\end{array}\right] .
$$

$\mathbf{R}$ is called the operational matrix of the section as $\int_{0}^{t} u(x, T) k(x, T) d T$ in terms of Chebyshev polynomials.

Therefore the initial equation is transformed into the products of several dependent matrixes as follows:

$$
\begin{gathered}
\boldsymbol{\Phi}^{T}(x) \operatorname{UAMA}^{T} \mathbf{G} \boldsymbol{\Phi}(x)+\boldsymbol{\Phi}^{T}(x) \mathbf{U P} \boldsymbol{\Phi}(t) \\
+\boldsymbol{\Phi}^{T}(x) \mathbf{U A V}_{(n+1) \times n} \mathbf{B}^{*} \boldsymbol{\Phi}(t) \\
+\boldsymbol{\Phi}^{T}(x) \mathbf{U A R A}^{T} \mathbf{K} \boldsymbol{\Phi}(x)=f(x, t) .
\end{gathered}
$$

Dispersing (48) with $\left(x_{i}, t_{j}\right)(i=1,2, \ldots, n ; j=1,2, \ldots, n)$, by using a symbolic software such as "Mathematica," we can get $\mathbf{U}$. So the numerical solution of the original problem is obtained ultimately.

\section{Numerical Examples}

To demonstrate the efficiency and the practicability of the proposed method based on Chebyshev polynomials method, we present some examples and find their solution via the method described in the previous section.

Example 1. Consider

$$
\begin{aligned}
D^{t / 3}[ & u(x, t)(x+t+1)]+\frac{\partial u(x, t)}{\partial t}+\int_{0}^{t} u(x, T) d T \\
& +\int_{0}^{t} u(x, T)(x+t) d T=f(x, t) \\
& u(x, 0)=x^{2} u(0, t)=t^{2}[x, t] \in[0,1] \times[0,1],
\end{aligned}
$$




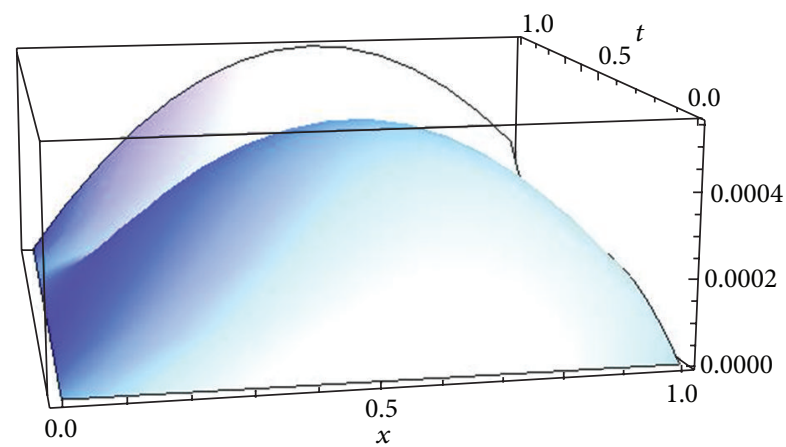

FIGURE 1: The absolute error between the numerical solution and the exact solution when $n=2$.

where

$$
\begin{aligned}
f(x, t)= & 2 t+\frac{t^{3}}{3}+\frac{t^{4}}{4}+\frac{t^{3} x}{3}+t x^{2}+\frac{t^{2} x^{2}}{2}+t x^{3} \\
& -\frac{3 t^{1-t / 3}[6 t(9+8 t)-6(-9+t) t x]}{(-9+t)(-6+t)(-3+t) \Gamma(1-t / 3)} .
\end{aligned}
$$

The exact solution of the above equation is $u(x, t)=x^{2}+t^{2}$.

Taking $n=2$, dispersing $x_{i}=k_{i} / 3-1 / 6, t_{j}=k_{j} / 3-$ $1 / 6\left(k_{i}=1,2,3 ; k_{j}=1,2,3\right)$, we can get the matrix $\mathbf{U}$ as follows:

$$
\mathbf{U}=\left[\begin{array}{ccc}
0 & 0 & 1 \\
0 & 0.00217 & 0.99905 \\
1 & 1.00036 & 1.99974
\end{array}\right]
$$

The absolute error between the exact solution and the numerical solution is displayed in Figure 1.

Taking $n=3$, dispersing $x_{i}=k_{i} / 4-1 / 8, t_{j}=k_{j} / 4-$ $1 / 8\left(k_{i}=1,2,3,4 ; k_{j}=1,2,3,4\right)$, we can get the matrix $\mathbf{U}$ as follows:

$$
\mathbf{U}=\left[\begin{array}{cccc}
0 & 0 & \frac{1}{3} & 1 \\
0 & 0 & \frac{1}{3} & 1 \\
\frac{1}{3} & \frac{1}{3} & \frac{2}{3} & \frac{4}{3} \\
1 & 1 & \frac{4}{3} & 2
\end{array}\right]
$$

The absolute error between the exact solution and the numerical solution is displayed in Figure 2.

Example 2. Consider

$$
\begin{aligned}
& D^{\sin t / 3}[u(x, t)(x t)]+\frac{\partial u(x, t)}{\partial t}+\int_{0}^{t} u(x, T) d T \\
& \quad+\int_{0}^{t} u(x, T)(x+t) d T=f(x, t) \\
& u(x, 0)=(1+x)^{2} u(0, t)=(1+t)^{2}[x, t] \in[0,1] \times[0,1],
\end{aligned}
$$

where

$$
\begin{aligned}
f(x, t)= & 2(1+x+t)+t+\frac{3 t^{2}}{2}+t^{3}+\frac{t^{4}}{4}+3 t x+3 t^{2} x+t^{3} x+3 t x^{2}+\frac{3 t^{2} x^{2}}{2}+t x^{3} \\
& -\frac{3 t^{1-\sin t / 3} x\left[54(1+t+x)^{2}+(1+x) \sin t(-3(5+4 t+5 x)+(1+x) \sin t)\right]}{\Gamma(1-\sin t / 3)(-9+\sin t)(-6+\sin t)(-3+\sin t)} .
\end{aligned}
$$

The exact solution of the above problem is $u(x, t)=(1+x+t)^{2}$.

Taking $n=2$, dispersing $x_{i}=k_{i} / 3-1 / 6, t_{j}=k_{j} / 3-$ $1 / 6(i=1,2,3 ; j=1,2,3)$, we can obtain the matrix $\mathbf{U}$ as follows:

$$
\mathbf{U}=\left[\begin{array}{ccc}
1 & 2 & 4 \\
2 & 3.50385 & 5.99903 \\
4 & 6.00062 & 8.99959
\end{array}\right]
$$

The absolute error between the exact solution and the numerical solution is displayed in Table 1 .

Taking $n=3$, dispersing $x_{i}=k_{i} / 3-1 / 6, t_{j}=k_{j} / 3-$ $1 / 6(i=1,2,3 ; j=1,2,3)$, the matrix $\mathbf{U}$ is displayed as follows:

$$
\mathbf{U}=\left[\begin{array}{cccc}
1 & \frac{5}{3} & \frac{8}{3} & 4 \\
\frac{5}{3} & \frac{23}{9} & \frac{34}{9} & \frac{16}{3} \\
\frac{8}{3} & \frac{34}{9} & \frac{47}{9} & 7 \\
4 & \frac{16}{3} & 7 & 9
\end{array}\right]
$$

The absolute errorbetween the exact solution and the numerical solution is displayed in Table 2 .

Taking $n=4$, dispersing $x_{i}=k_{i} / 5-1 / 10, t_{j}=$ $k_{j} / 5-1 / 10(i=1,2, \ldots, 5 ; j=1,2, \ldots, 5)$, the matrix $\mathbf{U}$ is displayed as follows: 
TABLE 1: The absolute error between the numerical solution and the exact solution when $n=2$.

\begin{tabular}{lccccc}
\hline & $t=0.1$ & $t=0.3$ & $t=0.5$ & $t=0.7$ & $t=0.9$ \\
\hline$x=0.0$ & 0 & 0 & 0 & 0 & 0 \\
$x=0.1$ & $2.2157 e-004$ & $4.8610 e-004$ & $5.1546 e-004$ & $2.8785 e-004$ & $1.5781 e-004$ \\
$x=0.2$ & $3.9341 e-004$ & $8.7893 e-004$ & $9.8541 e-004$ & $5.3056 e-004$ & $2.9527 e-004$ \\
$x=0.3$ & $5.5481 e-004$ & $1.3762 e-003$ & $1.7659 e-003$ & $7.9111 e-004$ & $3.5739 e-004$ \\
$x=0.4$ & $6.0429 e-004$ & $1.6415 e-003$ & $1.7751 e-003$ & $8.4552 e-004$ & $4.1018 e-004$ \\
$x=0.5$ & $6.5633 e-004$ & $1.6016 e-003$ & $1.6325 e-003$ & $8.8767 e-004$ & $4.3163 e-004$ \\
$x=0.6$ & $6.3392 e-004$ & $1.4087 e-003$ & $1.6103 e-003$ & $9.1437 e-004$ & $3.8174 e-004$ \\
$x=0.7$ & $5.6511 e-004$ & $1.2886 e-003$ & $1.5435 e-003$ & $8.6002 e-004$ & $2.9051 e-004$ \\
$x=0.8$ & $4.9215 e-004$ & $1.2345 e-003$ & $1.2001 e-003$ & $7.5921 e-004$ & $1.7393 e-004$ \\
$x=0.9$ & $3.8106 e-004$ & $7.8650 e-004$ & $8.754 e-004$ & $5.5526 e-004$ & $1.3122 e-005$ \\
$x=1.0$ & $1.8274 e-004$ & $3.8541 e-004$ & $4.3935 e-003$ & $3.8035 e-004$ & $1.9023 e-004$ \\
\hline
\end{tabular}

TABLE 2: The absolute error between the numerical solution and the exact solution when $n=3$.

\begin{tabular}{lccccr}
\hline & $t=0.1$ & $t=0.3$ & $t=0.5$ & $t=0.7$ & 0 \\
\hline$x=0.0$ & 0 & 0 & 0 & $t=0.9$ \\
$x=0.1$ & $1.87636 e-015$ & $2.43249 e-015$ & $4.37659 e-016$ & $3.19872 e-015$ & 0 \\
$x=0.2$ & $2.01345 e-015$ & $2.58254 e-015$ & $1.39627 e-015$ & $5.14303 e-015$ & $7.08487 e-015$ \\
$x=0.3$ & $2.67658 e-015$ & $3.92180 e-015$ & $4.47129 e-016$ & $4.56189 e-015$ & $5.18520 e-015$ \\
$x=0.4$ & $1.79236 e-015$ & $2.62554 e-015$ & $4.72489 e-016$ & $5.64307 e-015$ & $5.34316 e-015$ \\
$x=0.5$ & $1.67836 e-015$ & $2.82354 e-015$ & $4.93589 e-016$ & $1.37827 e-015$ & $3.34771 e-015$ \\
$x=0.6$ & $1.56727 e-015$ & $3.10922 e-015$ & $8.60978 e-016$ & $1.93427 e-015$ & $8.28178 e-015$ \\
$x=0.7$ & $2.17645 e-015$ & $1.77326 e-015$ & $1.39936 e-016$ & $3.18642 e-015$ & $2.26454 e-015$ \\
$x=0.8$ & $8.73278 e-016$ & $2.82354 e-015$ & 0 & $2.63545 e-015$ & $4.24089 e-016$ \\
$x=0.9$ & $4.56389 e-016$ & $1.93236 e-015$ & $8.65478 e-016$ & $4.62389 e-015$ & $5.20703 e-015$ \\
$x=1.0$ & $1.80036 e-015$ & $2.79454 e-015$ & $8.44378 e-016$ & $2.76054 e-015$ & $5.70912 e-015$ \\
\hline
\end{tabular}

$$
\mathbf{U}=\left[\begin{array}{ccccc}
1 & \frac{3}{2} & \frac{13}{6} & 3 & 4 \\
\frac{3}{2} & 2.125 & 2.91667 & \frac{31}{8} & 5 \\
\frac{13}{6} & 2.91667 & 3.83333 & 4.91667 & 6.1667 \\
3 & 3.875 & 4.91667 & 6.125 & 7.5 \\
4 & 5 & \frac{37}{6} & 7.5 & 9
\end{array}\right]
$$

The absolute error between the exact solution and the numerical solution is displayed in Table 3.

When $g(x, t)=u(x, t)$, the initial equation becomes nonlinear equation. Example 3 describes the situation.

Example 3. Consider

$$
\begin{aligned}
D^{t / 3} u^{2}(x, t) & +D^{t / 4} u(x, t)+\frac{\partial^{2} u(x, t)}{\partial t^{2}}=f(x, t) \\
u(x, 0) & =x^{2}, u(0, t)=t^{2}(x, t) \in[0,1] \times[0,1],
\end{aligned}
$$

where

$$
\begin{gathered}
f(x, t) \\
=2
\end{gathered}
$$

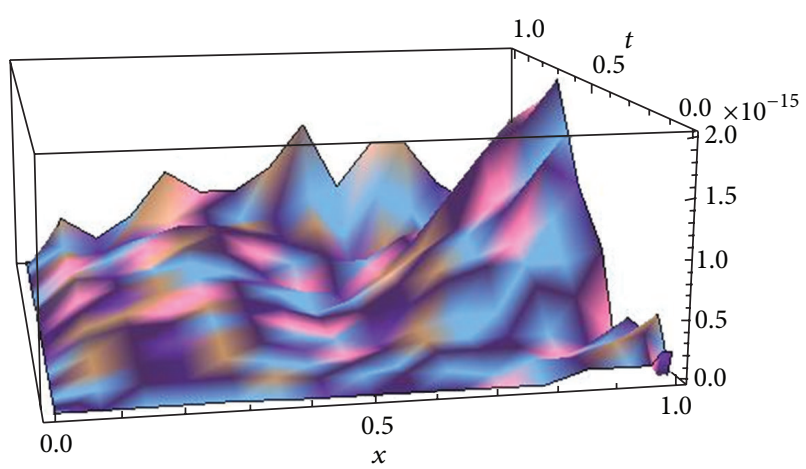

Figure 2: The absolute error between the numerical solution and the exact solution when $n=3$.

$$
\begin{aligned}
& +\frac{36 t^{2-t / 3}\left(54 t^{2}+(-12+t)(-9+t) x^{2}\right)}{(-12+t)(-9+t)(-6+t)(-3+t) \Gamma(1-t / 3)} \\
& +\frac{32 t^{2-t / 4}}{\left(32-12 t+t^{2}\right) \Gamma(1-t / 4)} .
\end{aligned}
$$

The exact solution of the above equation is $u(x, t)=x^{2}+t^{2}$. 
TABLE 3: The absolute error between the numerical solution and the exact solution when $n=4$.

\begin{tabular}{lccccc}
\hline & $t=0.1$ & $t=0.3$ & $t=0.5$ & $t=0.7$ & 0 \\
\hline$x=0.0$ & 0 & 0 & 0 & 0.9 \\
$x=0.1$ & $4.23485 e-015$ & $2.53645 e-016$ & $3.67471 e-015$ & $1.34255 e-014$ & $1.84674 e-014$ \\
$x=0.2$ & $2.65749 e-015$ & $4.43989 e-016$ & $2.30435 e-015$ & $1.25053 e-014$ & $5.96979 e-015$ \\
$x=0.3$ & $2.34298 e-016$ & $1.83436 e-015$ & $3.34257 e-015$ & $1.80056 e-015$ & $1.34590 e-014$ \\
$x=0.4$ & $3.37542 e-015$ & $1.65736 e-015$ & $1.43257 e-015$ & $1.56836 e-015$ & $1.57838 e-014$ \\
$x=0.5$ & $2.39005 e-015$ & $3.12362 e-015$ & $1.56436 e-015$ & $3.45782 e-015$ & $1.10141 e-014$ \\
$x=0.6$ & $1.29456 e-015$ & $2.64564 e-015$ & $1.45637 e-015$ & $4.87698 e-015$ & $1.43013 e-014$ \\
$x=0.7$ & $1.69936 e-015$ & $4.56239 e-016$ & $3.56342 e-015$ & $4.59329 e-015$ & $4.56750 e-014$ \\
$x=0.8$ & $5.42507 e-015$ & $2.76554 e-015$ & $2.34525 e-015$ & $1.58931 e-014$ & $5.97889 e-014$ \\
$x=0.9$ & $7.86761 e-015$ & $7.89961 e-015$ & $3.27652 e-015$ & $3.31246 e-014$ & $4.14391 e-014$ \\
$x=1.0$ & $7.18553 e-015$ & $1.54690 e-014$ & 0 & $5.45820 e-014$ & $5.56484 e-014$ \\
\hline
\end{tabular}

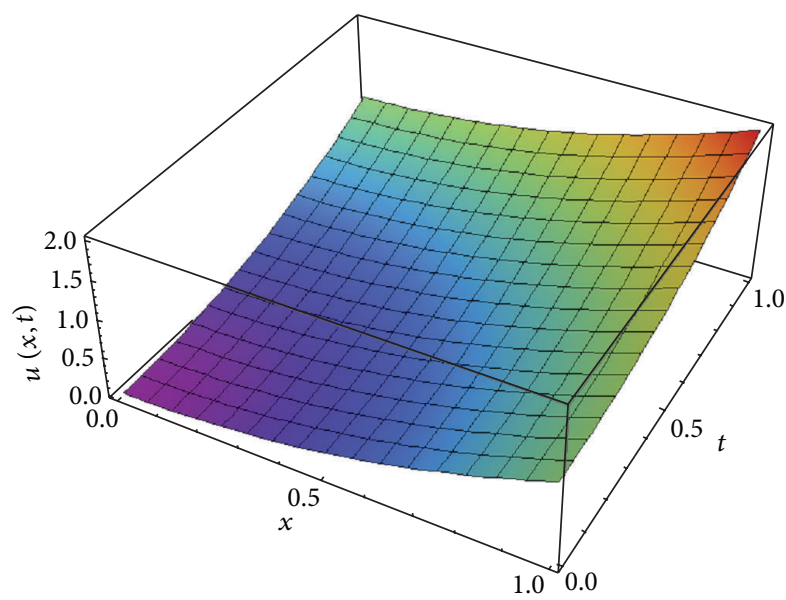

FIGURE 3: The numerical solution for Example 3 of $n=2$.

This is a nonlinear variable order fractional differential equation; the numerical solution can also be gained with the method proposed in Section 3 when $n \leq 2$.

Taking $n=2$, dispersing $x_{i}=k_{i} / 2-1 / 4, t_{j}=k_{j} / 2-$ $1 / 4\left(k_{i}=1,2 ; k_{j}=1,2\right)$, we can obtain the matrix $\mathbf{U}$ as follows:

$$
\mathbf{U}=\left[\begin{array}{lll}
0 & 0 & 1 \\
0 & 0 & 1 \\
1 & 1 & 2
\end{array}\right]
$$

The numerical solution obtained by our method and the exact solution are shown in Figures 3 and 4. The absolute error between the exact solution and the numerical solution is displayed in Figure 5.

When $n \geq 3$, the computation is very large and getting the numerical solution is a very difficult thing.

From Figures 1-5, Tables 1-3, we can see that the absolute errors are very small and only a small number of Chebyshev polynomials are needed. Compared with the other methods proposed in $[19,20]$, the method in this paper has significant advantages. The calculating results also show that combined with Chebyshev polynomials the method in this paper can

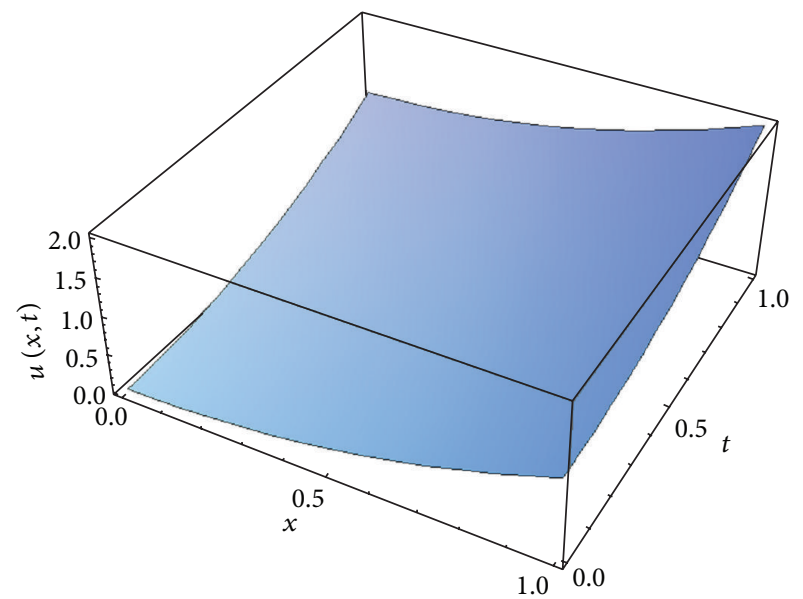

FIgURE 4: The exact solution for Example 3.

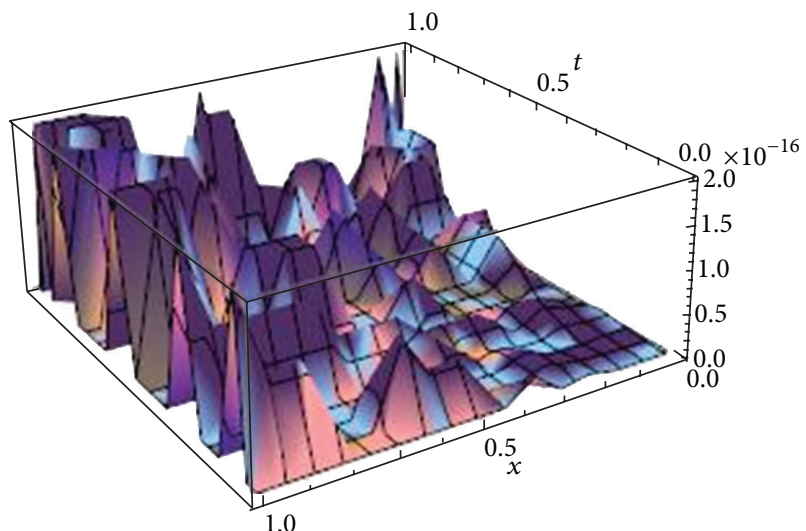

Figure 5: The absolute error for Example 3 of $n=2$.

be effectively used in the numerical solution of the fractional equation. From the above results, the numerical solutions are in good agreement with the exact solution. 


\section{Conclusion}

In the present paper, the application and scope of the Chebyshev polynomials have been extended to a class of variable order fractional integral-differential equation successfully. Actually we derive four kinds of operational matrixes using Chebyshev polynomials and use these to solve the variable order fractional integral-differential equation numerically. By solving the system of algebraic equations, numerical solutions are obtained. Numerical examples illustrate the powerfulness of the proposed method. The solutions obtained using the suggested method show that numerical solutions are in very good coincidence with the exact solution. The method can be applied by developing for the other fractional problem.

\section{Conflict of Interests}

The authors declare that there is no conflict of interests regarding the publication of this paper.

\section{Acknowledgment}

This work was supported by the National Natural Science Foundation of China under Grant no. 51307004.

\section{References}

[1] J. C. Wang, "Realizations of generalized Warburg impedance with RC ladder networks and transmission lines," Journal of the Electrochemical Society, vol. 134, no. 8, pp. 1915-1920, 1987.

[2] F. J. Valdes-Parada, J. A. Ochoa-Tapia, and J. Alvarez-Ramirez, "Effective medium equations for fractional Fick's law in porous media," Physica A: Statistical Mechanics and its Applications, vol. 373, pp. 339-353, 2007.

[3] H. Sun, W. Chen, C. Li, and Y. Chen, "Fractional differential models for anomalous diffusion," Physica A: Statistical Mechanics and Its Applications, vol. 389, no. 14, pp. 2719-2724, 2010.

[4] M. Ichise, Y. Nagayanagi, and T. Kojima, "An analog simulation of non-integer order transfer functions for analysis of electrode processes," Journal of Electroanalytical Chemistry, vol. 33, no. 2, pp. 253-265, 1971.

[5] H. H. Sun, A. A. Abdelwahab, and B. Onaral, "Linear approximation of transfer function with a pole of fractional order," IEEE Transactions on Automatic Control, vol. 29, no. 5, pp. 441-444, 1984.

[6] R. C. Koeller, "Applications of fractional calculus to the theory of viscoelasticity," Journal of Applied Mechanics, vol. 51, no. 2, pp. 299-307, 1984.

[7] Z. M. Odibat, "A study on the convergence of variational iteration method," Mathematical and Computer Modelling, vol. 51, no. 9-10, pp. 1181-1192, 2010.

[8] I. L. El-Kalla, "Convergence of the Adomian method applied to a class of nonlinear integral equations," Applied Mathematics Letters, vol. 21, no. 4, pp. 372-376, 2008.

[9] M. M. Hosseini, "Adomian decomposition method for solution of nonlinear differential algebraic equations," Applied Mathematics and Computation, vol. 181, no. 2, pp. 1737-1744, 2006.

[10] S. Momani, Z. Odibat, and V. S. Erturk, "Generalized differential transform method for solving a space- and time-fractional diffusion-wave equation," Physics Letters A, vol. 370, no. 5-6, pp. 379-387, 2007.

[11] Z. Odibat, S. Momani, and V.S. Erturk, "Generalized differential transform method: application to differential equations of fractional order," Applied Mathematics and Computation, vol. 197, no. 2, pp. 467-477, 2008.

[12] Y. M. Chen, M. X. Yi, and C. X. Yu, "Error analysis for numerical solution of fractional differential equation by Haar wavelets method," Journal of Computational Science, vol. 3, no. 5, pp. 367373, 2012.

[13] J. L. Wu, "A wavelet operational method for solving fractional partial differential equations numerically," Applied Mathematics and Computation, vol. 214, no. 1, pp. 31-40, 2009.

[14] C. F. Lorenzo and T. T. Hartley, "Variable order and distributed order fractional operators," Nonlinear Dynamics, vol. 29, no. 14, pp. 57-98, 2002.

[15] C. F. Coimbra, "Mechanics with variable-order differential operators," Annalen der Physik, vol. 12, no. 11-12, pp. 692-703, 2003.

[16] M. A. Snyder, Chebyshev Methods in Numerical Approximation, Prentice-Hall, Englewood Cliffs, NJ, USA, 1966.

[17] E. H. Doha, A. H. Bhrawy, and S. S. Ezz-Eldien, "A Chebyshev spectral method based on operational matrix for initial and boundary value problems of fractional order," Computers and Mathematics with Applications, vol. 62, no. 5, pp. 2364-2373, 2011.

[18] S. G. Samko, "Fractional integration and differentiation of variable order," Analysis Mathematica, vol. 21, no. 3, pp. 213-236, 1995.

[19] R. Lin, F. Liu, V. Anh, and I. Turner, "Stability and convergence of a new explicit finite-difference approximation for the variable-order nonlinear fractional diffusion equation," Applied Mathematics and Computation, vol. 212, no. 2, pp. 435-445, 2009.

[20] P. Zhuang, F. Liu, V. Anh, and I. Turner, "Numerical methods for the variable-order fractional advection-diffusion equation with a nonlinear source term," SIAM Journal on Numerical Analysis, vol. 47, no. 3, pp. 1760-1781, 2009. 


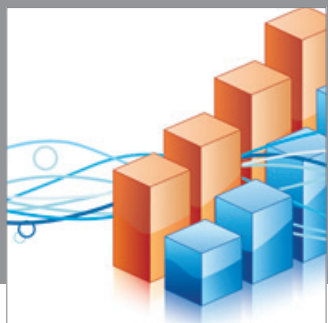

Advances in

Operations Research

mansans

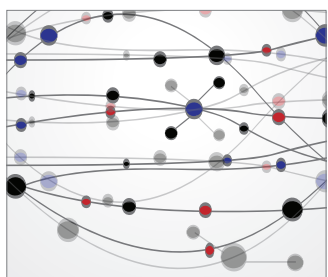

The Scientific World Journal
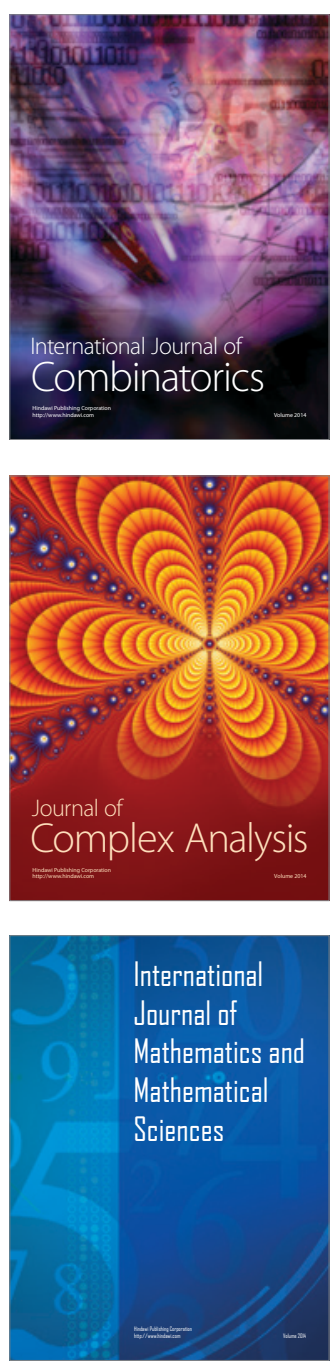
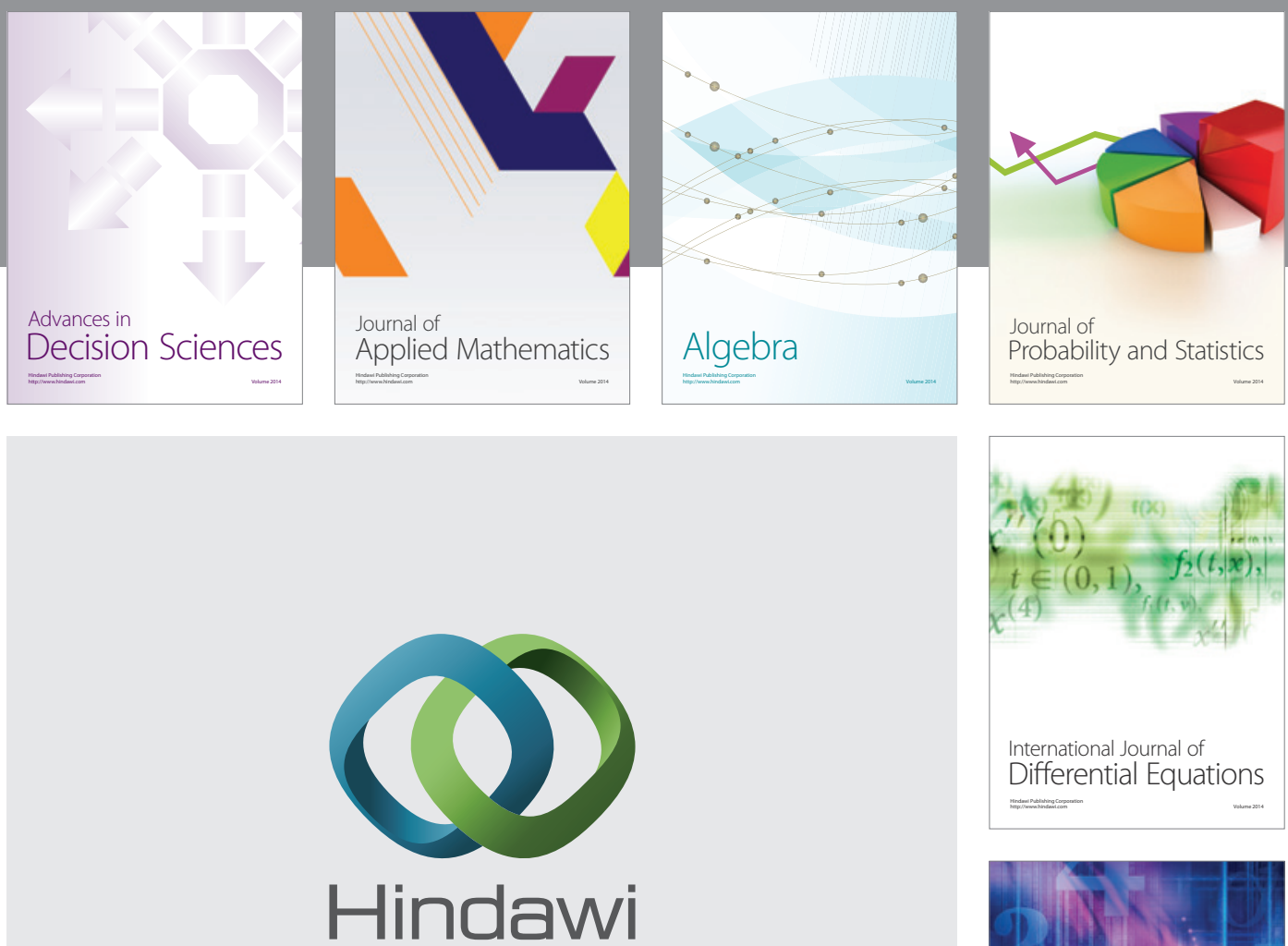

Submit your manuscripts at http://www.hindawi.com
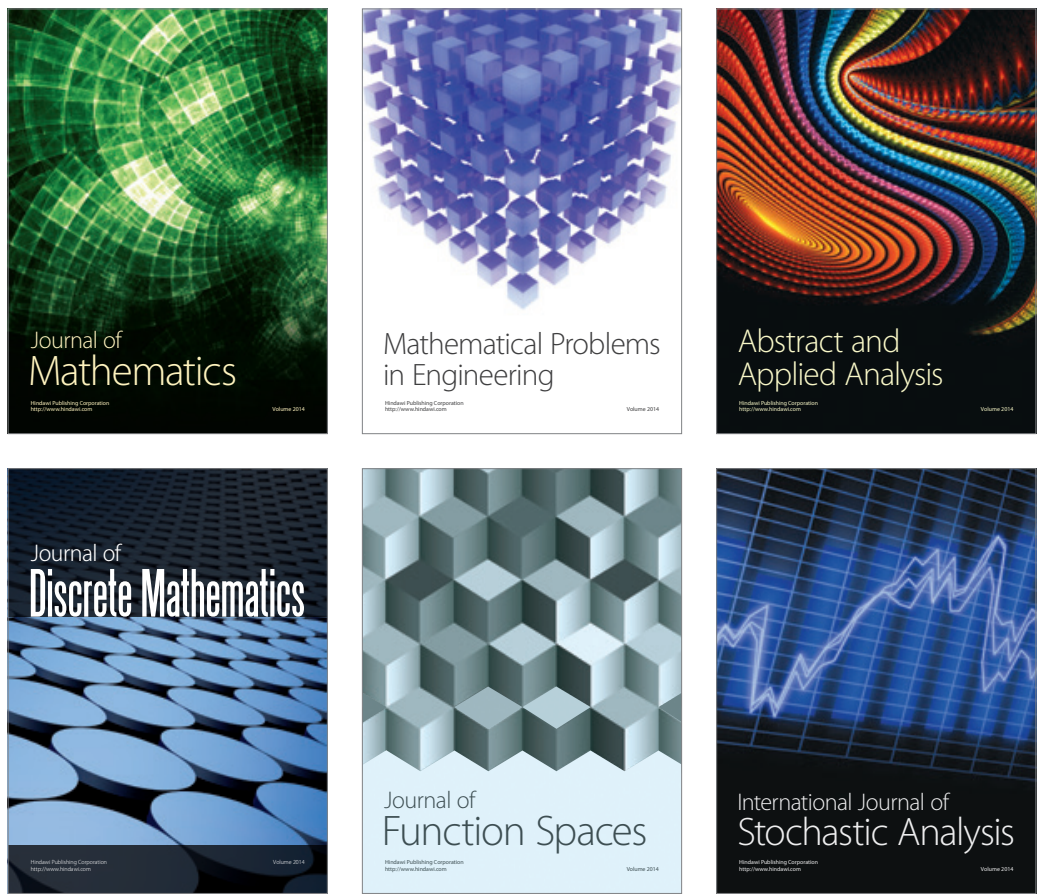

Journal of

Function Spaces

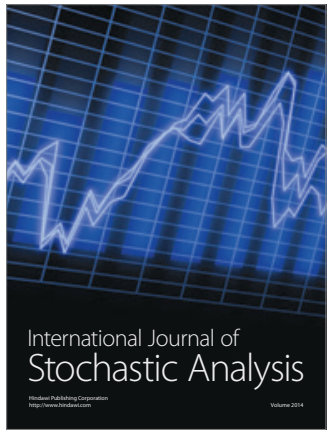

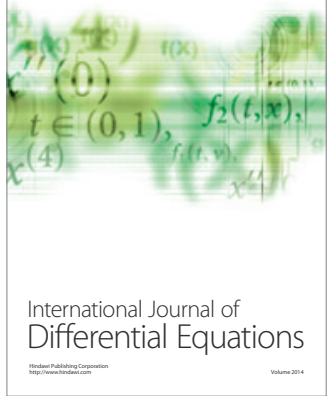
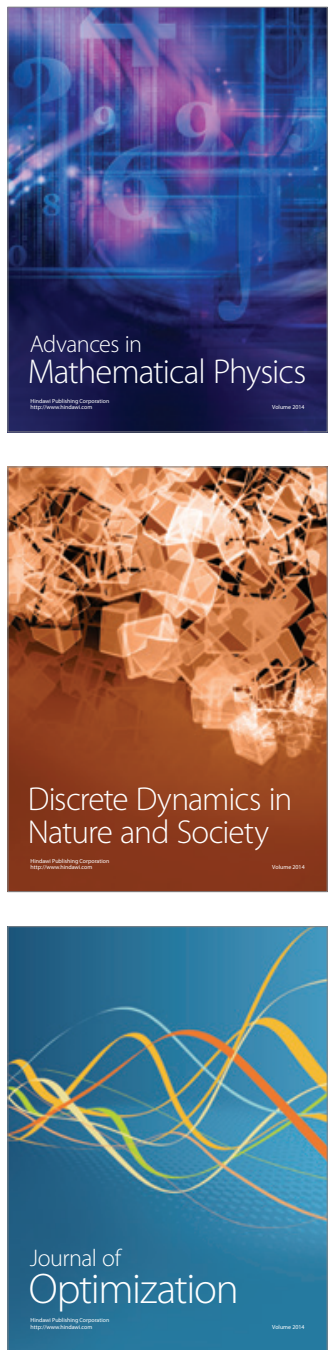